Dabei bedeutet $\chi_{0}=-\frac{1}{3} \cdot\left(\mu^{2} n\right) / k T$ die konstante diamagnetische Suszeptibilität pro Volumeneinheit der freien Elektronen im Magnetfeld.

Die Formel (59) zeigt eine Verminderung der Konstante der diamagnetischen Suszeptibilität der freien Elektronen im Magnetfeld durch den Einfluß des Gitterpotentials.

9 A. Janussis, Phys. Status Solidi 7, 765 [1964].
Eine ähnliche Formel wie (59) ist vom Autor ${ }^{9}$ in einer früheren Arbeit entwickelt.

Aus der Formel (51) zeigt sich wieder, daß die Singularitäten der Zustandssumme, die für die Berechnung des HaAs-van Alphen-Effektes ${ }^{10}$ erforderlich sind, genau an der Stelle der freien Elektronen auftreten und unabhängig von der Bindung des Festkörpers sind.

10 A. H. Wilson, Proc. Cambridge Phil. Soc. 49, 292 [1953].

\title{
Näherungsansätze zum Ising-Modell nach einer neueren Diagrammentwicklung I
}

\author{
H. Hartmann und H. P. Neumann \\ Institut für Physikalische Chemie der Universität Frankfurt am Main \\ (Z. Naturforschg. 22 a, $604-612$ [1967] ; eingegangen am 17. September 196)
}

\begin{abstract}
Die „Selbstenergien“ $G_{n}$ in ${ }^{1}$ wurden durch näherungsweise Auswertung einer größeren Klasse von Selbstenergiediagrammen approximativ berechnet. Das Gleichungssystem (3.3) in 1 für die renormierten Semiinvarianten wurde umgeformt und durch zusätzliche Näherungsannahmen vereinfacht. Durch Näherungsansätze für die Semiinvarianten $M_{2}, M_{3}, \ldots$ konnten einfache Gleichungen für die Magnetisierung $M_{1}$ hergeleitet werden. Diese Gleichungen wurden numerisch gelöst. Auf der Grundlage der Beziehungen (3.5) und (4.8) in ${ }^{1}$ wurden ferner die innere Energie, die freie Energie und die Atomwärme des zweidimensionalen Ising-Ferromagneten sowie die Druck-Dichte-Isothermen des zweidimensionalen Gittergases numerisch ausgerechnet.
\end{abstract}

In einer Serie moderner Arbeiten ${ }^{1-3}$ wurde eine ältere Reihenentwicklung ${ }^{4}$ der freien Energie $f_{\mathrm{c}}$ des Ising-Modells nach dem Parameter $\beta=1 / k T(T=$ absolute Temperatur) mit THieleschen Semiinvarianten als Koeffizienten zu einer Clusterentwicklung aller wichtigen thermodynamischen Funktionen des IsingModells ausgebaut. Besonders in ${ }^{1}$ wird ausführlich dargelegt, wie sich die Magnetisierung $M_{1}$, die innere Energie $\varepsilon$ und die freie Energie $f_{\mathrm{c}}$ als folgende Funktionen der Selbstenergien $G_{n}$ und der renormierten Semiinvarianten $M_{n}$ darstellen lassen:

$$
\begin{aligned}
& M_{1}=\exp \left[\sum_{K=1} G_{K} \frac{\partial K}{\partial x^{K}}\right] M_{1}^{0}(x), \\
& M_{n}=\exp \left[\sum_{K=1} G_{K} \frac{\partial K}{\partial x^{K}}\right] M_{n}^{0}(x)
\end{aligned}
$$

mit

$$
\begin{gathered}
M_{n}{ }^{0}(x)=\frac{\mathrm{d}^{n}}{\mathrm{~d} x^{n}}[\ln (2 \cosh x)], n=0,1,2, \ldots, \\
-\varepsilon=\frac{1}{2 \beta}\left[\sum_{n=1}^{\infty} n M_{n} G_{n}\right] \\
-\beta f_{\mathrm{c}}=M_{0}-\sum_{n=1}^{\infty} M_{n} G_{n}+\sum_{n=1}^{\infty} \frac{1}{2} \int_{0}^{1} \frac{\mathrm{d} \xi}{\xi} n M_{n} G_{n} .
\end{gathered}
$$

1 F. Englert, Phsy. Rev. 129, 567 [1963].

2 R. Brout u. M. Coopersmth, Phys. Rev. 130, 253 [1963].
Eine Selbstenergie $G_{n}$ ist dabei die Summe aller vertexirreduziblen Diagramme, die mit $n$ Linien an einem Fixpunkt aufgehängt sind. Jedem Diagramm entspricht ein Produkt der Form

$$
\left(\beta v_{i j}\right)^{l_{1}}\left(\beta v_{k h}\right)^{l_{2}} \ldots\left(M_{m_{1}}(x)\right)^{r_{1}} \cdot\left(M_{m_{2}}(x)\right)^{r_{2}} \ldots
$$

$\left(v_{i j}=\right.$ Wechselwirkung zwischen Spin $i$ und Spin $j$, $\beta=1 / k T, x=\beta H, H=$ Magnetfeldstärke). Jeder Linie im Diagramm korrespondiert ein Faktor $\beta v_{i j}$ und jedem Schnittpunkt von $l$ Linien eine Semiinvariante $M_{l}(x)$.

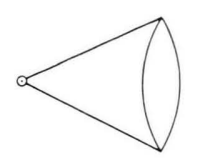

Abb. 1. Selbstenergiediagramm aus $G_{22}$.

Jedes Diagramm mit $n$ Linien und dem topologischen Index $t$ erhält noch einen Symmetriefaktor $\mathrm{l} / G(n, t)$, wobei $G(n, t)$ die Ordnungszahl der vollen Symmetriegruppe des Graphs ist.

Die Selbstenergien $G_{n}$ sind also Funktionen der renormierten Semiinvarianten $M_{n}$.

3 H. B. Callen u. G. Horwitz, Phys. Rev. 124, 1757 [1961].

${ }^{4}$ J. G. Kirkwood, J. Chem. Phys. 6, 70 [1938]. 
Mit $M_{1}$ und $f_{\mathrm{c}}$ sind auch die Druck-Dichte- $(p-\varrho)$ Isothermen bekannt, denn es gelten die Beziehungen

$$
\begin{gathered}
\varrho(H)=\left(1-M_{1}(H)\right) / 2, \\
p(H)=-\underset{\text { Isisg }}{\left(f_{\mathrm{c}}(H)+v(0) / 2+H\right) .} .
\end{gathered}
$$

Eine Lösung des fundamentalen Gleichungssystems ( 1,1 a) setzt nun voraus, daß die funktionale $\mathrm{Ab}$ hängigkeit $\operatorname{der} G_{n}$ von den $M_{K}$ zumindest approximativ bekannt ist. $G_{1}$ ist exakt gleich $\beta v(0) M_{1}$

$$
\left(v(0)=\sum_{i \neq j=1}^{V} v_{i j}\right) \text {. }
$$

$G_{2}$ wurde bisher durch Summation aller Ringdiagramme mit einem Fixvertex approximiert.

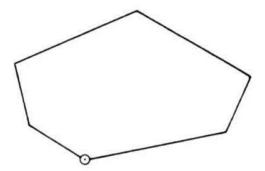

Abb. 2. Ringdiagramm mit Fixvertex.

Im nächsten Abschnitt werden alle $G_{n}$ bis zu beliebig hoher Ordnung $n$ durch eine Approximation, die derjenigen von $G_{2}$ ähnelt, angenähert. In den darauf folgenden Abschnitten werden Näherungsmethoden zur Berechnung von $M_{1}, f_{\mathrm{c}}, \varepsilon, p$ und $\varrho$ auf der Grundlage der Gln. (1) - (6) aufgestellt und numerische Ergebnisse angegeben.

\section{Näherungsweise Berechnung der Selbstenergien}

\section{a) Allgemeine Beziehungen}

An Stelle von Ringen aus einfachen Bindungen, wie sie zur Berechnung von $G_{2}$ benutzt wurden, sollen im allgemeinen Fall $G_{n}$ nur Ringe aus Büscheln von einfachen Bindungen summiert werden, wobei die Knoten der Büschelringe Schnittpunkte von jeweils $n$ einfachen Bindungen sind.

Es steht dabei die Erwartung im Hintergrund, daß die $G_{n}$ durch die Büschelringe in gleicher Güte angenähert werden wie $G_{2}$ durch die einfachen Ringe.

Bezeichnet man die Fourier-Transformierte von Bündeln aus $v$ einfachen Bindungen und $r$ Bündeln den Beitrag

$$
\begin{aligned}
& \sum_{\substack{\delta_{1},-\delta_{n} \\
\left(\delta_{1}+\ldots+\delta_{2 r}=0\right)}} s(n, v) \frac{\left(\beta v_{\delta_{1}}\right)^{v}}{v !} \underset{\left(n-v v_{\delta_{2}}\right)^{n-v}}{(n-v) !} \cdots \frac{\left(\beta v_{\delta_{2} r-1}\right)^{v}}{v !} \frac{\left(\beta v_{\delta_{2} r}\right) n^{n-v}}{(n-v) !}\left(M_{n}\right)^{2 r-1} \\
& =\frac{1}{V} s(n, v) \sum_{\boldsymbol{q}}\left(\frac{\beta^{v} M_{n} v^{(v)}(\boldsymbol{q}) \beta^{n-v} M_{n} v^{(n-v)}(\boldsymbol{q})}{v !} \frac{1}{M_{n}}\right) .
\end{aligned}
$$


$\boldsymbol{\delta}_{\mu}$ sind hierbei Abstandsvektoren zwischen zwei Gitterpunkten des Ising-Modells. Die Summation $\Sigma$ wird über die erste Brillouinsche Zone des reziproken Gitters geführt. $s(n, v)$ ist ein Symmetriefaktor. Er ist 1 für $v \neq n-v$ und $\mathrm{l} / 2$ für $v=n-v=\lambda$. $V$ ist die Anzahl der Gitterplätze.

Mit Hilfe von (9) erhält man durch Summation über alle $r=1,2, \ldots, \infty$ für $\Phi_{v}{ }^{(n)}$ und $\Psi_{\lambda}^{(n)}$ die Ausdrücke:

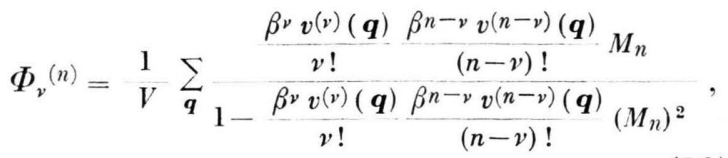

$$
\psi_{\lambda}^{(n)}=\frac{1}{2 V} \sum_{\boldsymbol{q}} \frac{\left(\frac{\beta^{\lambda} v^{(\lambda)}(\boldsymbol{q})}{\lambda !}\right)^{2} M_{n}}{1-\frac{\beta^{\lambda} v^{(\lambda)}(\boldsymbol{q})}{\lambda !} M_{n}} .
$$

Damit sind nach (7) und (8) alle $G_{n}$ bestimmt.

b) Approximative Auswertung der $\Phi_{v}^{(n)}$ und $\Psi_{i}{ }^{(n)}$ mit dem speziellen Potential ${ }^{5} v_{i j}=\exp \left[-\gamma{\left.r_{i j}{ }^{2}\right]}\right]$

Die Summationen über die diskreten Parameter in (9), (10) und (11) sind recht schwerfällige Operationen. Es ist deshalb zweckmäßig, sie näherungsweise durch Integrationen über unendlich große Integrationsbereiche zu ersetzen. Dies ist sicher erlaubt, da die mittleren Atomabstände im Kristall

sehr klein sind. Andererseits soll der Kristall aus statistisch-thermodynamischen Gründen sehr groß sein. (9), (10) und (11) ändern sich dann entsprechend ab in die Beziehungen:

$$
\begin{aligned}
& \int_{\substack{\infty \\
\delta_{1}+\delta_{2}+\ldots+\delta_{2 r}=0}}^{\infty} \mathrm{d}^{s} \delta_{1} \ldots \int_{-\infty}^{\infty} \mathrm{d}^{s} \delta_{2 r} s(n, v) \stackrel{\left(\beta v_{\delta_{1}}\right)^{v}}{v !} \quad \underset{(n-v) !}{\left(\beta \delta_{\delta_{2}}\right)^{n-v}} \times \ldots \times \frac{\left(\beta v_{\delta_{n-1}}\right)^{v}}{v !} \times \frac{\left(\beta v_{\delta_{n}}\right)^{n-v}}{(n-v) !}\left(M_{n}\right)^{2 r-1} \\
& =s(n, v) \frac{1}{(2 \pi)^{s}} \int_{-\infty}^{\infty}\left(\begin{array}{c}
\beta^{v} M_{n} v^{(v)}(\boldsymbol{q}) \cdot 2 \pi \\
v !
\end{array}\right)^{r}\left(\frac{\beta^{n-v} \cdot M_{n} v^{(n-v)}(\boldsymbol{q}) \cdot 2 \pi}{(n-v) !}\right)^{r} \cdot \frac{1}{M_{n}} \mathrm{~d}^{s} \boldsymbol{q},
\end{aligned}
$$

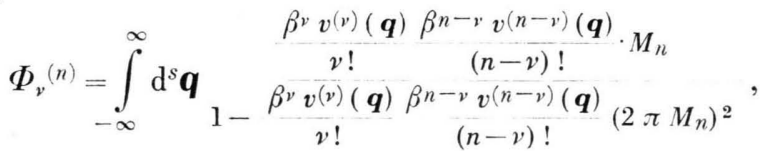

$$
\begin{aligned}
& \Psi_{\lambda}{ }_{\lambda}^{(n)}=\int_{-\infty}^{\infty} \mathrm{d}^{s} \boldsymbol{q} \underset{1-\frac{\beta^{\lambda} \frac{v^{(\lambda)}(\boldsymbol{q})}{\lambda !} v^{(\lambda)}(\boldsymbol{q})}{\lambda !} 2 \pi M_{n}}{\left(\frac{\beta_{n}}{\lambda !}\right.}
\end{aligned}
$$

mit

$v^{(\nu)}(\boldsymbol{q})=\frac{1}{(2 \pi)^{s / 2}} \int_{-\infty}^{\infty} \mathrm{d} \delta_{1} \ldots \int_{-\infty}^{\infty} \mathrm{d} \delta_{s} \exp \left[-v \gamma\left(\delta_{1}{ }^{2}+\ldots+\delta_{s}{ }^{2}\right)\right] \cdot \exp \left[i\left(q_{1} \delta_{1}+\ldots+q_{s} \delta_{s}\right)\right]$

( $s$ ist die Dimension des Gitters des IsınG-Modells. Es sollen hier die beiden Fälle $s=2,3$ betrachtet werden).

Das Integral (15) ist geschlossen ausführbar. Es ergibt sich:

$$
v^{(v)}(\boldsymbol{q})=\frac{1}{(2 \gamma v)^{s / 2}} \exp \left[-\left(q_{1}^{2}+\ldots+q_{s}^{2}\right) / 4 \gamma v\right] .
$$

Einsetzen von (16) in (13) und (14) führt auf die Integrale

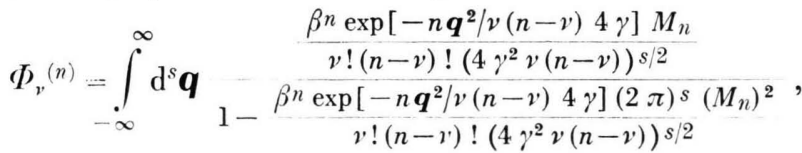

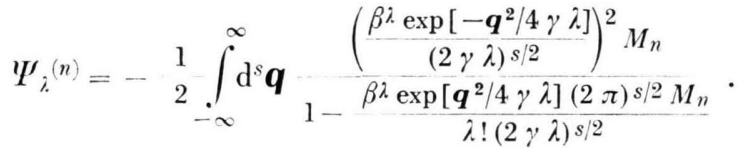

5, , ist eine Konstante. 
Beim zweidimensionalen Problem $(s=2)$ sind die Integrale (17) und (18) geschlossen integrierbar. Man erhält die Formeln:

$$
\begin{aligned}
& \Phi_{\nu}^{(n)}=-\frac{\gamma \nu(n-v)}{\pi n M_{n}} \ln \left[1-\frac{\beta^{n}\left(M_{n}\right)^{2} \pi^{2}}{\nu !(n-\nu) ! \gamma^{2} v(n-v)}\right], \\
& \Psi_{\lambda}^{(n)}=-\frac{\beta \lambda}{2 \lambda !}\left[1+\frac{\lambda ! \gamma \lambda}{\beta \lambda \pi M_{n}} \ln \left[1-\frac{\beta^{\lambda} \pi M_{n}}{\gamma \lambda \lambda !}\right]\right] .
\end{aligned}
$$

Im dreidimensionalen Fall $(s=3)$ kann (17) und(18) auf folgende eindimensionale Integrale reduziert werden:

$$
\begin{gathered}
\Phi_{\nu}^{(n)}=\frac{4 \pi \gamma v(n-v)}{(2 \pi)^{3} n M_{n}} \int_{-\infty}^{\infty} \mathrm{d} q_{3} \ln \left[1-\frac{(2 \pi)^{3} \beta^{n}\left(M_{n}\right)^{2}}{\nu !(n-\nu) !\left(4 \gamma^{2} \nu(n-v)\right)^{3 / 2}} \cdot \exp \left[-n q_{3}^{2} / 4 \gamma \nu(n-v)\right]\right], \\
\Psi_{\lambda}^{(n)=-} \frac{\gamma \lambda}{(2 \pi)^{2} M_{n}} \int_{-\infty}^{\infty} \mathrm{d} q_{3}\left[\left(\frac{\pi}{\gamma \lambda}\right)^{3 / 2} \frac{\beta \lambda}{\lambda !} M_{n} \exp \left[-q_{3}{ }^{2} / 4 \gamma \lambda\right]+\ln \left(1-\left(\frac{\pi}{\gamma \lambda}\right)^{3 / 2} \frac{\beta^{2}}{\lambda !} M_{n} \exp \left[-q_{3}{ }^{2} / 4 \gamma \lambda\right]\right)\right] .
\end{gathered}
$$

Nimmt man nun an, daß die renormierten Semiinvarianten $M_{2}, M_{3}, \ldots$ kleine Korrekturgrößen sind (Faktor $\times M_{n} \ll 1$ ), so kann man (19), (20), (21) und (22) in eine TAyLoR-Reihe entwickeln und nach dem ersten Glied abbrechen. (19) - (22) vereinfachen sich dann erheblich. Man erhält für das zweidimensionale Modell

$$
\begin{aligned}
& \Phi_{\nu}{ }^{(n)}=\frac{\beta^{n} \pi M_{n}}{\gamma \nu !(n-\nu) ! n}, \\
& \Psi_{\lambda}{ }^{(n)}=\frac{1}{2} \frac{\beta^{n} \pi M_{n}}{\gamma(\lambda !)^{2} n} .
\end{aligned}
$$

Für das dreidimensionale Modell ergibt sich:

$$
\begin{aligned}
& \Phi_{\nu}^{(n)}=\left(\frac{\pi}{\gamma n}\right)^{3 / 2} \frac{\beta^{n} M_{n}}{v !(n-\nu) !}, \\
& \Psi_{\lambda}^{(n)}=\left(\frac{\pi}{\gamma n}\right)^{3 / 2} \frac{\beta^{n} M_{n}}{(\lambda !)^{2}} .
\end{aligned}
$$

Mit den Ausdrücken (23) - (26) lassen sich in (7) und (8) die Summen mit Hilfe des binomischen Satzes leicht ausrechnen. Für die Selbstenergien $G_{n}$ resultieren so im zweidimensionalen Fall die Formeln:

$$
\begin{aligned}
G_{2 \lambda+1} & =\sum_{\nu=1}^{\lambda} \Phi_{\nu}{ }^{(2 \lambda+1)} \\
& =\frac{\pi \beta^{2 \lambda+1} M_{2 \lambda+1}}{\gamma(2 \lambda+1)} \sum_{\nu=1}^{\lambda} v !(2 \lambda+1-v) ! \\
& =\frac{\pi \beta^{2 \lambda+1} M_{2 \lambda+1}}{\gamma(2 \lambda+1) \cdot 2(2 \lambda+1) !}\left(\sum_{\nu=0}^{2 \lambda+1} \frac{(2 \lambda+1) !}{\nu !(2 \lambda+1-\nu) !}-2\right) \\
G_{2 \lambda+1} & =\frac{\pi \beta^{2 \lambda+1} M_{2 \lambda+1}}{\gamma(2 \lambda+1)(2 \lambda+1) !}\left(\frac{1}{2} 2^{2 \lambda+1}-1\right), \quad \lambda=1,2,3, \ldots, \\
\text { ebenso } & \lambda
\end{aligned}
$$

Im dreidimensionalen Fall ergeben sich in analoger Weise für die Selbstenergien $G_{n}$ die Näherungsformeln:

$$
\begin{aligned}
G_{2 \lambda+1} & =\left(\frac{\pi}{\gamma(2 \lambda+1)}\right)^{3 / 2} \frac{\beta 2 \lambda+1 M_{2 \lambda+1}}{(2 \lambda+1) !}\left(\frac{1}{2} 2^{2 \lambda+1}-1\right), \\
G_{2 \lambda} & =\left(\frac{\pi}{\gamma \cdot 2 \lambda}\right)^{3 / 2} \frac{\beta^{2 \lambda} M_{2 \lambda}}{(2 \lambda) !}\left(\frac{1}{2} 2^{2 \lambda}-1\right), \quad \\
\lambda & \lambda=1,2,3, \ldots .
\end{aligned}
$$

\section{Umformung und Vereinfachung des Gleichungssystems (1) und (1 a)}

Die Gln. (1) und (1 a) mit Exponentialdifferentialoperatoren haben eine sehr unübersichtliche Form. Eine erste Maßnahme zu ihrer Vereinfachung ist die Diagonalisierung der Exponentialdifferentialoperatoren durch Fourier-Zerlegung von tanh $x$.

Für tanh $x$ existiert die Fourier-Darstellung ${ }^{6}$

$$
\begin{gathered}
M_{1}{ }^{0}(x)=\tanh x=\int_{-\infty}^{\infty} \tilde{M}_{1}(k) \exp [i k x] \mathrm{d} k \\
\tilde{M}_{1}(k)=\frac{1}{i} \operatorname{sign}(k) \frac{\exp [-\pi|k| / 2]}{1-\exp [-\pi|k|]}
\end{gathered}
$$

Damit gehen (1) und ( 1 a) über in das System

$$
\begin{gathered}
M_{1}(x)=\int_{\infty}^{\infty} \exp \left[\sum_{K=2}^{\infty} G_{K}\left(M_{2}, \ldots, M_{n}, \ldots\right)(i k)^{K}\right] \\
\tilde{M}_{1}(k) \exp [i k y] \mathrm{d} k \\
M_{n}(x)=\int_{-\infty}^{\infty} \exp \left[\sum_{K=2}^{\infty} G_{K}\left(M_{2}, \ldots, M_{n}, \ldots\right)(i k)^{K}\right] \\
\cdot(i k)^{n-1} \tilde{M}_{1}(k) \exp [i k y] \mathrm{d} k \quad(32 \mathrm{a}) \\
n=2,3,4, \ldots,
\end{gathered}
$$


wobei $y$ die Funktion

$$
y=\beta v(0) M_{1}(x)+x
$$

ist.

Man hat nun mit (32) und (32 a) immer noch ein unendliches Gleichungssystem für die unendlich vielen unbekannten Funktionen $M_{1}(x), M_{2}(x), \ldots$, vorliegen. Wie in ${ }^{1}$ gezeigt wurde, hat nur die Semiinvariante $M_{1}$ physikalische Bedeutung. $M_{1}$ ist nämlich identisch mit der Magnetisierung des IsingFerromagneten. Alle höheren Semiinvarianten sind nicht direkt physikalisch interpretierbar. Sie scheinen die Rolle von Hilfsgrößen zu spielen, und es wird zweckmäßig sein, sie zu eliminieren. Eine zentrale Stellung im Gleichungssystem (32), (32 a) nimmt die Funktion

$$
\dot{M}(k, y)=\exp \left[\sum_{K=2}^{\infty} G_{K}\left(M_{2}, \ldots, M_{n}, \ldots\right)(i k)^{K}\right]
$$

ein.

Mit ihrer Hilfe können alle Semiinvarianten in Integralform dargestellt werden.

Da die Selbstenergien $G_{2}, \ldots, G_{n}, \ldots$ noch funktional von den Semiinvarianten $M_{2}, \ldots, M_{n}, \ldots$ abhängen, ist $\tilde{M}(k, y)$ zunächst unbekannt.

Man erhält für $\tilde{M}(k, y)$ eine Integralgleichung, wenn man in (34) in den Selbstenergien $G_{K}$ die Semiinvarianten $M_{2}, \ldots, M_{n}, \ldots$ durch ihre Integraldarstellungen mit $\tilde{M}(k, y)$ als Kern beschreibt.

Führt man das Funktional

$$
\begin{aligned}
F_{k}(u, \tilde{M}(k, y), y)=\sum_{l=2}^{\infty} G_{l}\left(\int_{-\infty}^{\infty} \mathrm{d} k \tilde{M}(k, y)(i k) \tilde{M}_{1}(k) \exp [i k y], \ldots\right. \\
\\
\left.\quad \int_{-\infty}^{\infty} \tilde{M}(k, y)(i k)^{n-1} \tilde{M}_{1}(k) \exp [i k y] \mathrm{d} k, \ldots\right)(i u)^{l}
\end{aligned}
$$

ein, so folgt die Integralgleichung

$$
\tilde{M}(u, y)=\exp \left[F_{k}(u, \tilde{M}(k, y), y)\right]
$$

oder $\quad \ln [\tilde{M}(u, y)]=F_{k}(u, \tilde{M}(k, y), y)$.

Die allgemeine Integralgleichung (36) ist für eine rechnerische Behandlung noch sehr kompliziert. Auch ein Abbrechen der unendlichen Reihe $F(u, \tilde{M}, y)$, kombiniert mit einer Verwendung der Beziehungen (19), (20) bzw. (21), (22) für die näherungsweise Berechnung der $G_{n}$, schafft hier keine wesentliche Erleichterung.

Betrachtet man die Semiinvarianten $M_{2}, \ldots$, $M_{n}, \ldots$, wieder als kleine Korrekturgrößen wie im vorigen Abschnitt, was auf die Verwendung der Selbstenergien $G_{n}(27)$, (28) bzw. (29), (30) hinausläuft, so wird man auf eine einfachere Beziehung geführt. Es ergibt sich die Integralgleichung

$$
\ln [\tilde{M}(u, y)]=\int_{-\infty}^{\infty} F(u, k, y) \tilde{M}(k, y) \mathrm{d} k
$$

mit

$$
F(u, k, y)=\frac{\tilde{M}_{1}(k) \exp [i k y]}{i k} \sum_{l=2}^{\infty}(-u k \beta)^{l} a_{l} .
$$

Dabei wurde die Vertauschbarkeit der unendlichen Integration mit der unendlichen Summation in (35) vorausgesetzt. Wegen des Verlaufs von $\tilde{M}_{1}(k)$ ist diese Annahme wahrscheinlich richtig. Streng genommen müßte man sie jedoch an einer gefundenen Lösung nachträglich verifizieren.

Setzt man in (32) alle Semiinvarianten $M_{2}, \ldots$, $M_{n}, \ldots=0$, so verschwinden auch alle Selbstenergien $G_{2}, \ldots, G_{n}, \ldots$ und (32) reduziert sich auf die Magnetisierungsgleichung des $\mathrm{W}_{\text {EIssschen Modells }}$

$$
M_{1}=\tanh \left[\beta v(0) M_{1}+x\right] .
$$

Da das Weisssche Modell, wie aus dem Verlauf der Atomwärme ersichtlich ist, bis auf den kritischen Punkt zumindest qualitativ mit der Erfahrung in guter Übereinstimmung ist, erscheint die Annahme, daß die Semiinvarianten $M_{2}, \ldots, M_{n}, \ldots$ als kleine Korrekturgrößen aufzufassen sind, in gewissem Maße gerechtfertigt.

Im zweidimensionalen Fall kann man die unendliche Reihe (32 a) noch auf ein Integral transformieren. Setzt man für $a_{l}$ wegen (27) und (28) die Werte

$$
\frac{\pi}{\gamma}\left(\frac{1}{2} 2^{l}-1\right) l \cdot l !,
$$

so gilt

$$
\sum_{l=2}^{\infty}(-u k \beta)^{l} a_{l}=\frac{\pi}{\gamma}\left[\frac{1}{2} \sum_{l=2}^{\infty} \frac{(-u k \beta \cdot 2)^{l}}{l \cdot l !}-\sum_{l=2}^{\infty} \begin{array}{c}
(-u k \beta) l \\
l \cdot l !
\end{array}\right] .
$$


Ferner bestehen die Beziehungen

$$
\begin{gathered}
x \frac{\partial}{\partial x} \sum_{l=2}^{\infty} \frac{x^{l}}{l \cdot l !}=\sum_{l=2}^{\infty} \frac{x^{l}}{l !}=\exp [x]-x-1, \\
L(x)=\sum_{l=2}^{\infty} \frac{x^{l}}{l \cdot l !}=\int_{0}^{x} \frac{\exp \left[x^{\prime}\right]-x^{\prime}-1}{x^{\prime}} \mathrm{d} x^{\prime} .
\end{gathered}
$$

Mit Hilfe von (38), (39) und (40) erhält man schließlich für $F(u, k, y)$ in (37 a) die Formel

$$
F(u, k, y)=\frac{\pi \tilde{M}_{1}(k) \exp [i k y]}{\gamma i k}\left[\frac{1}{2} L(-2 u k \beta)-L(u k \beta)\right] .
$$

Die Integralgleichung (37) in Verbindung mit (41) ist immer noch verhältnismäßig kompliziert. Es erhebt sich daher die Frage, ob man nicht durch einfache Näherungsansätze für die renormierten Semiinvarianten $M_{2}, \ldots, M_{n}, \ldots$ zusammen mit den Näherungsausdrücken (27) - (30) für die Selbstenergien und den Gln. (1) bzw. (32) einfache Magnetisierungsgleichungen aufstellen kann, die gegenüber dem WeIssschen Modell verbesserte Lösungen aufzuweisen haben.

\section{Näherungsgleichungen für die Magnetisierung auf der Grundlage der Gln. (1) und (32)}

Der einfachste Ansatz für $M_{n}$ ist $M_{n}^{0}(y)$ mit $y=\beta v(0) M_{1}+x$. Er bedeutet, $\mathrm{da} \beta$ man in (1 a) und (32 a) alle $G_{K}$ mit $K \geqq 2$ vernachlässigt. Die $M_{n}{ }^{0}(y)$ haben aber noch nicht die Größenordnung von kleinen Korrekturgrößen. Dies kann man durch Multiplikation mit einer hinreichend kleinen Konstanten erreichen.

$$
\begin{aligned}
M_{n}(x) & =C_{n}(\beta) M_{n}^{0}(y), \\
y & =\beta v(0) M_{1}(x)+x .
\end{aligned}
$$

Verwendet man (42) zusammen mit (27), (28) bzw. (29), (30) in (32) und bricht man die unendliche Reihe $\sum_{K=2}^{\infty}$ an einer endlichen Stelle $l_{0}$ ab, so ergibt sich folgende approximierte Magnetisierungsgleichung:

$$
\begin{aligned}
M_{1}(x)= & \int_{-\infty}^{\infty} \mathrm{d} k \tilde{M}_{1}(k) \exp [i k y] \\
& \cdot \exp \left[\sum_{l=2}^{l_{0}} G_{l}\left(C_{l}(\beta) M_{l}^{0}(y)\right)(i k)^{l}\right], \\
y= & \beta v(0) M_{1}+x .
\end{aligned}
$$

Eine andere Möglichkeit entsteht, wenn man in (32) die Exponentialfunktion in eine Reihe entwickelt und mit dem linearen Glied abbricht. Man erhält dann wegen (2) die unendliche Reihe

$$
M_{1}(x)=\tanh y+\sum_{l=2}^{\infty} G_{l} M_{l+1}^{0}(y) .
$$

Ersetzt man in (44) die unendliche Reihe wieder durch ein Polynom und benutzt man ferner als Selbstenergien $G_{n}$ die $G_{n}$ aus (27), (28) bzw. (29), (30) in Verbindung mit dem Ansatz (42), so erhält man eine weitere approximierte Magnetisierungsgleichung

$$
\begin{gathered}
M_{1}(x)=\tanh y+\sum_{l=2}^{l_{0}} G_{l}\left(C_{l}(\beta) M_{l}^{0}(y)\right) M_{l+1}^{0}(y), \\
y=\beta v(0) M_{1}+x .
\end{gathered}
$$

Für Gln. (43) und (45) wurde für das zweidimensionale Problem je ein Beispiel auf einem Computer numerisch durchgerechnet. Für (43) wurde folgende Gleichung gewählt :

$$
\begin{gathered}
M_{1}(x)=\int_{-\infty}^{\infty} \mathrm{d} k \tilde{M}_{1}(k) \exp [i k y] \exp \left[\sum_{l=2}^{6} G_{l}(i k)^{l}\right], \\
y=\frac{\beta}{2 \gamma} M_{1}(x)+x
\end{gathered}
$$

mit

$$
\begin{gathered}
G_{1}=\beta v(0) M_{1}=\frac{\beta M_{1}}{2 \gamma}, \quad G_{2}=\frac{\pi \beta^{2} M_{2}}{\gamma}, \\
G_{3}=\frac{\pi \beta^{3} M_{3}}{6 \gamma}, \\
G_{4}=\frac{\pi \beta^{4} M_{4}}{24 \gamma}, \quad G_{5}=\frac{\pi \beta^{5} M_{5}}{40 \gamma}, \\
G_{6}=\frac{41 \pi \beta^{6} M_{6}}{432}
\end{gathered}
$$

und

$$
\begin{gathered}
M_{2}=C^{2} M_{2}{ }^{0} ; \quad M_{3}=C^{3} M_{3}{ }^{0} ; \quad M_{4}=-C^{4}\left|M_{4}{ }^{0}\right| ; \\
M_{5}=C^{5} y\left|M_{5}{ }^{0}\right| /|y| ; \quad M_{6}=C^{6}\left|M_{6}{ }^{0}\right|
\end{gathered}
$$

$\left[M_{2}{ }^{0}, \ldots, M_{6}{ }^{0}\right.$ wird nach Formel (2) berechnet].

Die Beträge in $M_{4}, \ldots, M_{6}$ mußten genommen werden, damit die Konvergenz des Integrals in (46) gewährleistet ist. Insofern weichen die Semiinvarian- 
ten in (48) etwas von dem Ansatz (42) ab. Als $C_{n}(\beta)$ wurden einfach die Potenzen $C^{n}$ der Konstanten $C$ genommen.

Durch geeignete Wahl von $C$ und $\gamma$ hat man noch die Möglichkeit, die Resultate besser an die empirischen Werte anzugleichen.

Als Beispiel für (45) wurde die Gleichung

$$
\begin{gathered}
M_{1}(x)=\tanh y+\sum_{l=2}^{3} G_{l} M^{0}{ }_{l+1}(y) \\
y=\frac{\beta}{2 \gamma} M_{1}(x)+x
\end{gathered}
$$

mit

$$
G_{2}=\frac{\pi \beta^{2} M_{2}}{2 \gamma} ; \quad G_{3}=\frac{\pi \beta^{3} M_{3}}{6 \gamma} ; \quad \begin{aligned}
& M_{2}=C^{2} M_{2}{ }^{0} ; \\
& M_{3}=C^{3} M_{3}{ }^{0}
\end{aligned}
$$

( $C=$ konstant $)$ numerisch gelöst.

Durch die Integration

$$
f_{\mathrm{c}}=-\int_{0}^{H} M_{1}\left(H^{\prime}\right) \mathrm{d} H^{\prime}+C_{\mathrm{int}}
$$

wurde aus den Lösungen von (46) und (49) die freie Energie numerisch bestimmt. Die Integrationskonstante $C_{\text {int }}$ in (50) wurde dabei durch die Bedingung

$$
\lim _{H \rightarrow \infty} f_{\mathrm{c}} \rightarrow-\left(\frac{1}{2} v(0)+H\right)
$$

festgelegt, was besagt, daß bei unendlich großen Feldstärken nur noch eine einzige Konfiguration (alle Spins in Richtung von $H$ ausgerichtet) praktisch vorliegt.

Mit Hilfe von (46), (49) und (50) wurden unter Benutzung von (5) und (6) die Druck-Dichte-Isothermen des Gittergases berechnet.

Als Grundlage zur Berechnung der inneren Energie diente Formel (3). Durch Verwendung der Lösungen von (46) und (49) in Verbindung mit (47) und (48) wurden nach (3) folgende Energieformeln ausgerechnet:

$$
\begin{gathered}
\varepsilon=-\frac{1}{2 \beta}\left[\frac{\beta M_{1}{ }^{2}}{2 \gamma}+\sum_{n=2}^{6} n C^{n} M_{n}{ }^{0}(y) G_{n}\right], \\
\varepsilon=-\begin{array}{c}
1 \\
2 \beta\left[\frac{\beta M_{1}{ }^{2}}{2 \gamma}+\sum_{n=2}^{3} n C^{n} M_{n}{ }^{0}(y) G_{n}\right], \\
y=\frac{\beta M_{1}}{2 \gamma}+x .
\end{array}
\end{gathered}
$$

Die Atomwärme $C_{u}$ wurde durch numerische Differentiation aus

bestimmt.

$$
C_{u}=-k \beta^{2}(\partial \varepsilon / \partial \beta)
$$

In den nachfolgend aufgeführten Tabellen werden einige repräsentative Werte angegeben. Wichtige Kurven sind in Abb. 4-Abb. 7 aufgetragen.

Die kritische Temperatur entsprechend (46) ergab sich durch Extrapolation zu

$$
\beta_{\mathrm{c}}=1 / k T_{\mathrm{c}}=0,24 .
$$

\begin{tabular}{clcl}
\hline$\beta$ & $M_{1}(0)$ & $\varepsilon(0)$ & $C_{u}(0) / k$ \\
\hline 0,02 & 0,0 & $-1,570 \cdot 10^{-6}$ & \\
0,06 & 0,0 & $-4,712 \cdot 10^{-6}$ & \\
0,10 & 0,0 & $-7,854 \cdot 10^{-5}$ & $7,854 \cdot 10^{-5}$ \\
0,14 & 0,0 & $-1,099 \cdot 10^{-5}$ & $1,539 \cdot 10^{-5}$ \\
0,18 & 0,0 & $-1,413 \cdot 10^{-5}$ & $2,500 \cdot 10^{-5}$ \\
0,22 & 0,0 & $-1,727 \cdot 10^{-5}$ & $6,650 \cdot 10^{-5}$ \\
\hline 0,26 & 0,6595 & $-1,087$ & 1,5305 \\
0,30 & 0,7982 & $-1,592$ & 0,8008 \\
0,34 & 0,8659 & $-1,874$ & 0,5875 \\
0,38 & 0,8999 & $-2,024$ & 0,3850 \\
0,42 & 0,9173 & $-2,103$ & \\
0,46 & 0,9268 & $-2,147$ & \\
\hline
\end{tabular}

Tab. 1. Magnetisierung, Energie und Atomwärme des zweidimensionalen Ising-Modells mit dem Potential $v_{i j}=e^{-\gamma r_{i j}{ }^{2}}$ und den Parameterwerten $H=0 ; C=0,1 ; \gamma=0,1$ nach Gl. (46), (51), (53).

\begin{tabular}{clcl}
\hline$\beta$ & $M_{1}(0)$ & $\varepsilon(0)$ & \multicolumn{1}{c}{$C_{u}(0) / k$} \\
\hline 0,02 & 0,0 & $-7,853 \cdot 10^{-6}$ & \\
0,06 & 0,0 & $-2,356 \cdot 10^{-5}$ & $1,413 \cdot 10^{-6}$ \\
0,10 & 0,0 & $-3,926 \cdot 10^{-5}$ & $3,926 \cdot 10^{-6}$ \\
0,14 & 0,0 & $-5,497 \cdot 10^{-5}$ & $7,696 \cdot 10^{-6}$ \\
0,18 & 0,0 & $-7,068 \cdot 10^{-5}$ & $1,270 \cdot 10^{-5}$ \\
& & & \\
0,22 & 0,7962 & $-0,615$ & 1,368 \\
0,26 & 0,7434 & $-1,381$ & 0,960 \\
0,30 & 0,8530 & $-1,819$ & 0,742 \\
0,34 & 0,9113 & $-2,076$ & 0,569 \\
0,38 & 0,9448 & $-2,232$ & \\
\hline
\end{tabular}

Tab. 2. Magnetisierung, Energie und Atomwärme des zweidimensionalen IsING-Modells mit dem Potential $v_{i j}=e^{-\gamma r_{i j}{ }^{*}}$ und den Parameterwerten $H=0 ; C=0,1 ; \gamma=0,1$ nach $\mathrm{Gl}$. (49), (52), (53).

Für die kritische Temperatur nach (49) wurde der Wert

$$
\beta_{\mathrm{c}}=0,21
$$

extrapoliert. - Mit Hilfe von (6) ergab sich für den kritischen Druck

$$
P_{\mathrm{c}}=0,83 \text {. }
$$

\section{Diskussion}

Ein Vergleich der in Abb. 4 und Abb. 5 aufgetragenen Kurven mit den Ergebnissen des exakten 


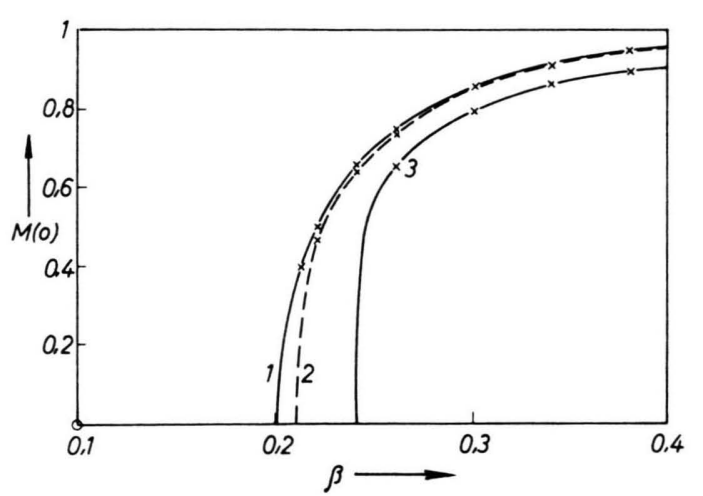

Abb. 4. Spontane Magnetisierungen des zweidimensionalen

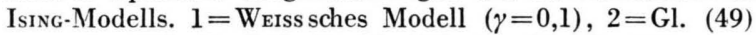
$(\gamma=0,1 ; C=0,1), 3=\mathrm{Gl}$. (46) $(\gamma=0,1 ; C=0,1)$.

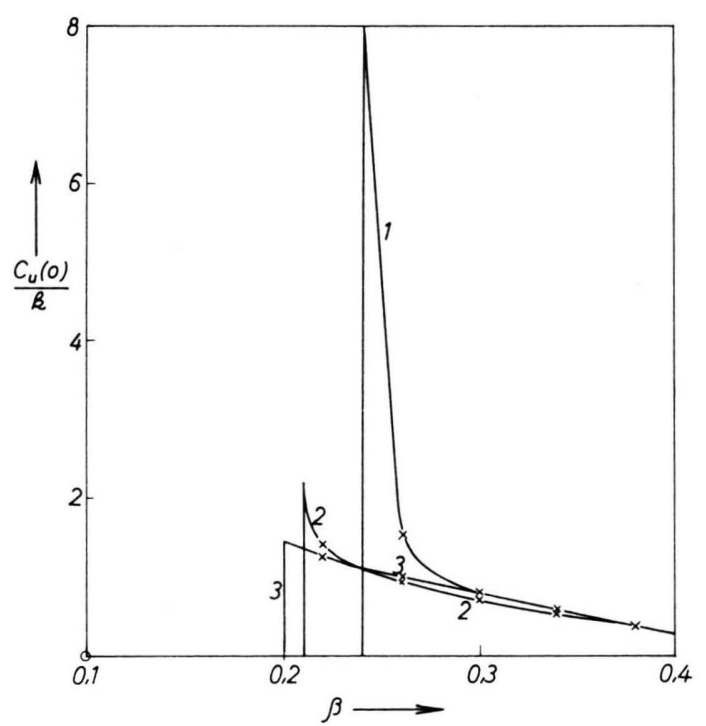

Abb. 5. Atomwärmen des zweidimensionalen Ising-Modells $(H=0) . \quad 1=\mathrm{Gl}$. (46) und (51) $(\gamma=0,1 ; C=0,1), 2=\mathrm{Gl}$. (49) und (52) $(\gamma=0,1 ; C=0,1), 3=\mathrm{W}_{\text {EIss sches Modell }}$ $(\gamma=0,1)$.

zweidimensionalen Ising-Modells in 7 , Seite 596, 599, (578) zeigt, daß schon die relativ einfachen approximierten Magnetisierungsgleichungen (46) und (49) in Verbindung mit den Energierelationen (51) und (52) gegenüber dem WeIssschen Modell bezüglich der Lage des kritischen Punktes und des Verlaufs der Atomwärmen im unterkritischen Bereich $\mathrm{zu}$ verbesserten Resultaten führen.

Mit Hilfe von (3) bzw. (51) und (52) ist aus (53) ersichtlich, daß ein steilerer Verlauf der spontanen Magnetisierung als Funktion von $\beta$ mit einem

7 A. Münster, Statistische Thermodynamik, Berlin 1956.

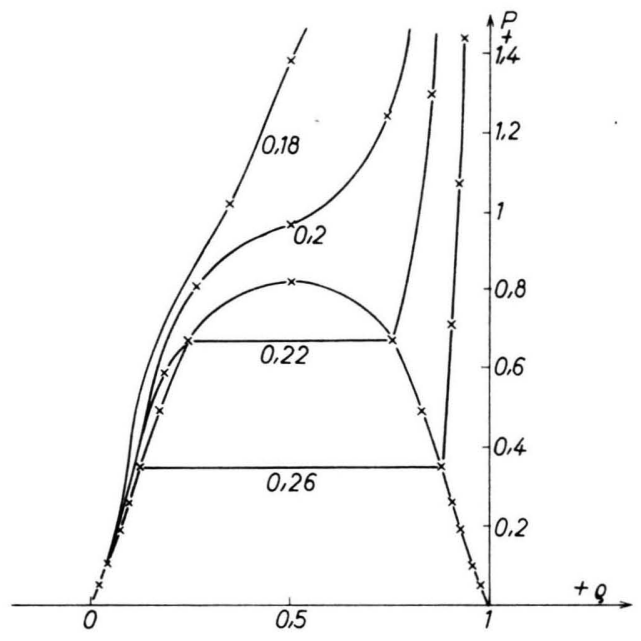

Abb. 6. Druck-Dichte-Isothermen des zweidimensionalen Gittergases errechnet aus (49), (50) und (6) $(\gamma=0,1 ; C=0,1)$.

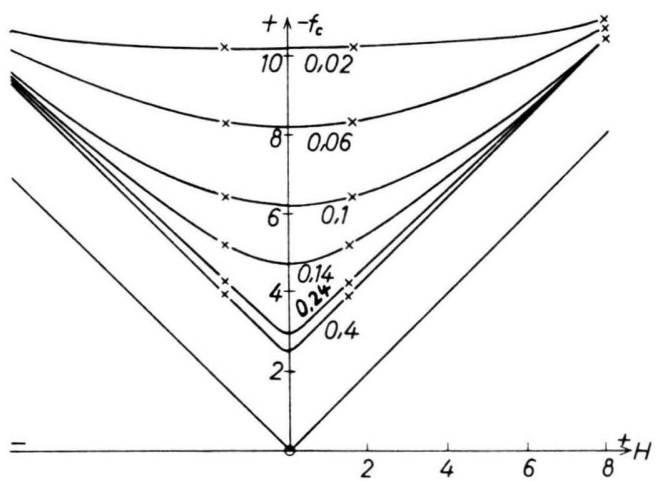

Abb. 7. Freie Energie des zweidimensionalen Ising-Modells nach (49) und (50) $(\gamma=0,1 ; C=0,1)$.

stärkeren Anwachsen der Atomwärme $C_{u}$ im unterkritischen Bereich der engeren Umgebung des kritischen Punktes zusammenhängt. Durch die Näherungen (46), (51) bzw. (49), (52) wird, wie die Zahlenwerte der Atomwärmen in Tab. 1 und Tab. 2 anzeigen, im überkritischen Gebiet eine Nahordnung beschrieben. Diese Nahordnung ist klein. Der Grund hierfür braucht jedoch nicht die speziell verwendete Näherung zu sein. Das Potential $v_{i j}=\exp \left[-\gamma r_{i j}{ }^{2}\right]$ mit dem Parameterwert $\gamma=0,1$ ist verhältnismäßig schwach und von langer Reichweite, so daß schon wegen dieser Tatsache keine große Nahordnung vorliegen kann.

Der Verlauf der Atomwärmen $C_{u}$ nach den Näherungen (46), (51) bzw. (49), (52) in unmittelbarer Umgebung des kritischen Punktes konnte durch die numerischen Rechnungen nicht genau bestimmt 
werden. Er wurde hier extrapoliert. Es ist möglich, daß die Atomwärmen $C_{u}$ am kritischen Punkt sogar unendlich groß werden. Neben dieser Ungewißheit ist das Verhalten der Atomwärmen in der unmittelbaren überkritischen Umgebung des kritischen Punktes etwas unbefriedigend. Aus (51) bzw. (52) und den Ansätzen für die Semiinvarianten (42), (48), (49) sieht man, daß die Atomwärmen beim Übergang vom überkritischen zum unterkritischen Gebiet einen Sprung haben, da in der Reihe (3) nur endlich viele Glieder berücksichtigt werden. Beim zweidimensionalen exakten Ising-Modell springt dagegen die Atomwärme nicht am kritischen Punkt, sondern wächst vom überkritischen Gebiet her dort stetig gegen unendlich.

Diese Diskrepanz rührt wahrscheinlich daher, daß in (51) bzw. (52) nicht alle Glieder von (3) berücksichtigt wurden, denn die Reihe (3) kann am kritischen Punkt $(y=0)$ konvergieren, während ihre Ableitung nach $\beta$ dort divergiert.

Es wird daher zum Studium der feineren Eigenschaften der Singularität der Atomwärme am kriti- schen Punkt notwendig sein, alle Selbstenergien und Semiinvarianten zumindest in ausreichender Näherung bei der unendlichen Summation zu berücksichtigen. Dazu gehört auch ein tieferer Einblick in die Struktur der Semiinvarianten $M_{2}, M_{3}, \ldots, M_{n}, \ldots$ zum Beispiel mit Hilfe der Integralgleichung (37).

Die Näherungsgleichungen (46), (51) liefern Ergebnisse, die der exakten Lösung noch näher kommen als die Resultate, die mit (49), (52) erzielt wurden. Dies liegt wahrscheinlich hauptsächlich daran, daß die Approximation des Exponentialoperators in (1) durch das lineare Glied eine zu grobe Näherung ist.

Die numerischen Rechnungen wurden auf der Datenverarbeitungsanlage vom System IBM 7090 des Deutschen Rechenzentrums in Darmstadt und auf dem Elektronenrechner Zuse Z 23 des Instituts für Physikalische Chemie der Universität Frankfurt am Main durchgeführt.

H. P. Neumann dankt der Deutschen Forschungsgemeinschaft für das ihm gewährte Stipendium. 